\title{
Metabolomic and transcriptomic analyses of Prunus davidiana reveal the key resistance compound to green peach aphid, betulin
}

\author{
Junxiu Wang
}

Zhengzhou Fruit Research Institute, Chinese Academy of Agricultural Sciences

\section{Yong Li}

Zhengzhou Fruit Research Institute, Chinese Academy of Agricultural Sciences

\section{Xinwei Wang}

Zhengzhou Fruit Research Institute, Chinese Academy of Agricultural Sciences

\section{$\mathrm{Ke} \mathrm{CaO}$}

Zhengzhou Fruit Research Institute, Chinese Academy of Agricultural Sciences

\section{Gengrui Zhu}

Zhengzhou Fruit Research Institute, Chinese Academy of Agricultural Sciences

\section{Weichao Fang}

Zhengzhou Fruit Research Institute, Chinese Academy of Agricultural Sciences

\section{Changwen Chen}

Zhengzhou Fruit Research Institute, Chinese Academy of Agricultural Sciences

\section{Jinlong Wu}

Zhengzhou Fruit Research Institute, Chinese Academy of Agricultural Sciences

\section{Jian Guo}

Zhengzhou Fruit Research Institute, Chinese Academy of Agricultural Sciences

\section{Qiang Xu}

College of Horticulture \& Forestry Sciences, Huazhong Agricultural University, Wuhan

Lirong Wang ( $\sim$ wanglirong@caas.cn )

Zhengzhou Fruit Research Institute, Chinese Academy of Agricultural Sciences

\section{Article}

Keywords: Betulin, Prunus davidiana, Myzus persicae, Metabolome, Transcriptome

Posted Date: March 2nd, 2021

DOI: https://doi.org/10.21203/rs.3.rs-258608/v1 
License: (c) (i) This work is licensed under a Creative Commons Attribution 4.0 International License. Read Full License 


\section{Abstract}

Prunus davidiana, a close wild relative of the cultivated peach, has been identified as having a strong resistance to Myzus persicae Sülzer (green peach aphid, GPA), one of the major pests of peach. However, the resistance mechanism of $P$. davidiana remains unclear. In this study, combined analysis of metabolome and transcriptome was conducted using aphid-resistant (R-32) and aphid-susceptible (S-27) lines, from a segregating population, to investigate the defense mechanism of $P$. davidiana. These results showed that R-32 continuously accumulated higher levels of betulin than S-27 during aphid infestation. Besides, betulin displayed a strong toxic effect on GPA. Transcriptome analysis revealed that the expression of genes involved in the betulin biosynthesis pathway responded highly to GPA infestation, especially CYP716A1. Our results demonstrate that betulin play an important role in the mechanism of resistance of $P$. davidiana to GPA.

\section{Introduction}

Crop wild relatives are abundant germplasm resources in nature and possess beneficial genes that are missing from their cultivated crops, especially resistance genes. The study of wild relative resistant germplasms with excellent agronomic traits can improve the understanding of the resistance of crops to environmental stress and promote the development of sustainable agriculture by reducing the use of chemicals ${ }^{1}$. Peach (Prunus persica (L.) Batsch) constantly encounters various unfavorable environments during its growth, including abiotic and biotic stresses. The green peach aphid (GPA, Myzus persicae Sülzer) is one of the major pests of peach, which not only affects its growth and production, but also mediates the spreading of a series of plant viruses. However, the gene that provides resistance to GPA is absent in the majority of currently cultivated peach varieties. Several aphid-resistant peach genotypes have been identified in previous studies. In the last century, two genotypes have been identified, namely "Weeping Flower Peach" (P. persica) and "Rubira" ( $P$. davidiana) ${ }^{2-4}$. Recently, Niu et al. have shown that "Fen Shouxing" (P. persica var. densa Makino) was also an aphid-resistant genotype and speculated that the WRKY DNA-binding protein gene (Prupe.1G564300) may be the gene that provides resistance ${ }^{5,6}$. However, the defense mechanism of $P$. davidiana, the variety with the strongest resistance among the 15 aphid-resistant germplasms in China ${ }^{7}$, is not clear.

P. davidiana, as a close wild relative of the cultivated peach, has been reported to have an antibiosis effect on GPA, which means that the fecundity of GPA decreased and the mean length of the mature embryos within the gonads of the females on the day of adult molt was negatively correlated with the total number of embryos ${ }^{8}$. Therefore, there are certain metabolites of $P$. davidiana that confer resistance to GPA. However, it is still not clear which metabolites of $P$. davidiana play a role in GPA resistance. The recently developed metabolomic technology has allowed the detection of metabolites at a highthroughput level. Several studies have identified metabolite changes during the infestation of Ostrinia furnacalis, Mythimna separata, Macrosiphoniella tanacetaria and Uroleucon tanaceti on maize and Tanacetum vulgare, respectively, using metabolomic technology ${ }^{9-11}$. However, the metabolomic changes 
during peach infestation by aphids remain unclear. Sauge ${ }^{12}$ showed that the major aphid-resistance quantitative trait loci (QTL, MP.SD-3.1), on G3 close to the AG106 and AG50B RFLP markers, was identified in $P$. davidiana. However, the key gene for GPA resistance in $P$. davidiana remains unclear.

Zhouxingshantao 1 , a representative germplasm of $P$. davidiana, shows strong resistance to aphids. In the present study, D29-32 (R-32) and D29-27 (S-27) from the segregating population of $P$. davidiana var. Zhouxingshantao 1 were resistant and susceptible, respectively, and were used to investigate the defense mechanism of $P$. davidiana. To unravel the underlying molecular defense mechanisms, we performed an integrated metabolomic and transcriptomic analysis of both R-32 and S-27 during aphid infestation to identify candidate key metabolites and genes that contribute to defense responses. This work provides a solid foundation for further research on the major genes responsible for resistance to GPA in P. davidiana.

\section{Results}

\section{The first two days post-infestation were the key period for the GPA response in $P$. davidiana}

Apterous GPAs were settled on the partially expanded leaves of five annual new shoots of resistant (R-32) and susceptible (S-27) individuals two weeks after spraying the chemical insecticide. The settlement and number of aphids per shoot were recorded every day for one week after aphid infestation (Fig. 1a, b). The number of aphids on R-32 slowly decreased and almost disappeared from 4 days post-infestation (DPI) and after. However, the aphid population on S-27 increased rapidly and gradually stabilized from 2 DPI and after. At the same time point, the number of aphids on S-27 was significantly higher than that on R$32(p<0.01)$. Therefore, we hypothesize that the resistance of R-32 to GPA is due to changes in metabolites in the first two days post infestation.

\section{Metabolomics landscape during GPA infestation in peach}

To understand the metabolomic changes during GPA infestation, the metabolites in R-32 and S-27 at 0, 6, 12,24 , and 48 hours post-infestation (hpi) were investigated using the UPLC-MS/MS platform (Wuhan Metware Biotechnology Co., Ltd., Wuhan, China). Finally, 449 known metabolites were identified, including 448 metabolites in R-32 and 446 metabolites in S-27. In R-32, 2, 3-Dihydroxypropyl-9, 12, 15octadecatrienoate-hexose-hexose was not detected, whereas betulin, quercetin 5-0-malonylhexosylhexoside, and quercetin 7-0-malonylhexosyl-hexoside were not identified in S-27. The 449 metabolites were clustered into 10 major categories $(P<0.05)$, including flavonoids $(86, \sim 19.15 \%)$, lipids $(67, \sim$ $14.92 \%)$, phenolic acids $(66, \sim 14.70 \%)$, amino acids and their derivatives $(56, \sim 12.47 \%)$, organic acids (37, 8.24\%), nucleotides and their derivatives (36, 8.02\%), lignans and coumarins $(18, \sim 4.01 \%)$, alkaloids $(17, \sim 3.79 \%)$, terpenes $(13, \sim 2.90 \%)$, and tannins $(2, \sim 0.45 \%)$ (Supplementary Table 1$)$. Several of these metabolites have been reported to play important roles in insect or disease resistance, for instance, flavonoids, phenolic acids, and alkaloids ${ }^{13-15}$.

The differentially accumulated metabolites (DAMs) ( $\log _{2} \mathrm{FC}$ (fold change) $\geq 1$ and VIP $\geq 1$ ) during GPA infestation (from 0 to $48 \mathrm{hpi}$ ) in resistant and susceptible accessions were identified by the comparison 
between GPA treatments and control (Fig. 1c). Finally, 192 and 217 DAMs were identified during GPA infestation in R-32 and S-27, respectively. Interestingly, the down-regulated DAMs were much more abundant than the up-regulated DAMs in both R-32 and S-27 in each treatment, suggesting that metabolites may participate in the response to GPA infestation via negative regulation. Notably, the changes in metabolite expression peaked at $6 \mathrm{hpi}$ in R-32 and at $12 \mathrm{hpi}$ in S-27, suggesting that the responses to aphid infestation in resistant accessions were faster than in susceptible accessions.

To study the changing trends of metabolite relative contents during GPA infestation, $K$-means cluster analysis was performed using Pearson correlation distances based on the expression pattern during GPA infestation. Finally, the 341 metabolites, with differences in at least two time points, could be classified into six major groups with 12 subclasses (Supplementary Fig. 1). Metabolites in group 1 (including 38 metabolites) was dominated by the flavonoids ( 47.37\%), harboring stable expression throughout the aphid infestation with the expression level in R-32 significantly higher than that in S-27. Group 2 could be divided into two subclasses, subclass 2 (34 metabolites) and subclass 3 (18 metabolites). Intriguingly, the expression of these metabolites peaked at $6 \mathrm{hpi}$ in R-32, while at $12 \mathrm{hpi}$ in S-27. The expression of metabolites in group 3, including subclass 4 (52 metabolites) and 5 (15 metabolites), showed different pattern in R-32 and S-27 lines, with a "delince-increase-decline" pattern in R-32 line and a "decline" pattern in S-27 line. In group 4, including subclass 6 (30 metabolites) and subclass 7 (19 metabolites), these metabolites were up-regulated at both $6 \mathrm{hpi}$ and $24 \mathrm{hpi}$ in R-32 and up-regulated at $12 \mathrm{hpi}$ in S-27. The metabolites in group 5 (76 metabolites) expressed stably in R-32 line but changed dramatically in S-27 line. In group 6, including three subclasses 10 (35 metabolites), 11 (9 metabolites), and 12 (15 metabolites), the expressions of these metabolites in R-32 were down-regulated at early stage of GAP infestation but up-regulated at late stage. For S-27, the lowest expression level was at $12 \mathrm{hpi}$ for subclass 10 and 11 , while the continuous increase was observed in subclass 12. Differences in metabolite expression during GPA infestation may be part of the defense mechanisms of $P$. davidianan var. Zhouxingshantao 1. From all six groups, it is interesting to observe that metabolites in Group 1 had higher expression levels in R-32 than in S-27 throughout the infestation by GAPs. These may be inherent differences between the two accessions, which include a category of metabolites that contribute to the constitutive defense mechanism.

\section{Candidate causative metabolites involved in GPA resistance}

To identify the possible causative metabolites for GPA resistance in peach, we compared the DAMs between R-32 and S-27 at all five time points (Fig. 2). In total, we identified 59, 81, 50, 68, and 61 upregulated DAMs in the comparison of R-32 and S-27 at 0, 6, 12, 24, and $48 \mathrm{hpi}$, respectively. Among these, 21 were shared between all the comparisons. Among the 21-shared DAMs, 19 metabolites belonged to subclass 1 , and two metabolites belonged to subclass 4 . Similarly, 152 downregulated DAMs were identified in the comparison of R-32 and S-27 during GPA infestation. Of these, 11 were shared at the five time points, including eight metabolites from subclass 9 and three metabolites from subclass 8 (Fig. 2a).

To further filter the representative metabolites in the GPA-resistant mechanism, we compared the top 20 up- and down- regulated DAMs between R-32 and S-27 at all five time points (Fig. 2b-2f). We found that 
betulin had the highest $\log _{2} \mathrm{FC}(>15)$ throughout the experiment, followed by quercetin 7-0malonylhexosyl-hexoside $\left(\log _{2} F C>13\right)$. Such high $\log _{2} \mathrm{FC}$ may be due to the very high levels of these two metabolites in R-32 compared to S-27. Although studies on the anti-insect effects of betulin are rare, betulin derivatives have been shown to have a strong effect against insects. Betulinic acid, an insect growth regulator, has been shown to effectively interfere with the growth of Spodoptera litura ${ }^{16}$ and Callosobruchus chinensis ${ }^{17}$. Therefore, it is reasonable to hypothesize that betulin, a lupane-type triterpene, may have an effect on GPAs. Quercetin, one of the most abundant flavonoids in plants, has been documented to be important in plant-insect interactions ${ }^{18-20}$. All these studies support our hypothesis that the quercetin derivative, quercetin 7-0-malonylhexosyl-hexoside, plays a role in the resistance to GPAs in P. davidiana.

\section{High toxicity of betulin to the GPA}

As glycosylation and malonylation of flavonoids increase their water solubility and stability ${ }^{21,22}$ we used quercetin instead of quercetin 7-0-malonylhexosyl-hexoside to verify its insecticidal toxicity. The toxicity of betulin and quercetin to GPA was determined using the leaf dipping method. The median lethal concentration $\left(\mathrm{LC}_{50}\right)$ values of betulin and quercetin after 12 and $24 \mathrm{~h}$ of exposure were further calculated (Fig. 3a). The results showed that $L C_{50}$ values of betulin and quercetin after $24 \mathrm{~h}$ of exposure were 396.08 and $727.56 \mathrm{mg} \cdot \mathrm{L}^{-1}$, respectively. After $48 \mathrm{~h}$ exposure, the $\mathrm{LC}_{50}$ values of betulin and quercetin were 206.64 and $306.34 \mathrm{mg} \cdot \mathrm{L}^{-1}$, respectively. This implies that betulin has a stronger toxic effect on GPA than quercetin. To further evaluate the inhibitory effect of betulin against aphids, we recorded the number of aphids on S-27 shoots after spraying with acetone (S-27 + acetone) or betulin (396.08 $\mathrm{mg} \cdot \mathrm{L}^{-1}, \mathrm{~S}-27+$ betulin) (Fig. 3b). This experiment showed a similar slow increase in the number of aphids on S-27+ acetone to that on S-27. Compared to S-27 + acetone, the number of aphids on S-27 + betulin remained at a lower level. After aphid infestation for 7 days, dead aphids were observed on S-27 + betulin (Fig. 3d). This indicated that betulin had an inhibitory effect on aphids. Moreover, the absolute content of betulin in R-32 (66.59 $\mu \mathrm{g} / \mathrm{g} \mathrm{DW})$ was significantly higher than that in S-27 (55.24 $\mu \mathrm{g} / \mathrm{g} \mathrm{DW}$ ) (Fig. 3c). Therefore, we suggest that betulin may play a crucial role in the resistance of $P$. davidiana to GPA.

\section{Transcriptomic changes during the GPA infestation in peach}

To elucidate the GPA defense mechanisms at the transcription level, RNA-seq was performed for R-32 and S-27 using the same samples used for the metabolite analyses. We generated 916 million clean reads using the Illumina platform. After aligning against the peach reference genome "Lovell" (release version $2.0)^{23}$, the average mapping rate was calculated to be $95.40 \%$ (Supplementary Table 2). Finally, 17, 813 genes expressed (FPKM $\triangle 1$ ) in at least one sample were identified, of which 13,923 were expressed in all samples (Supplementary Table 3). The RNA-seq results were further verified by qRT-PCR (Supplementary Fig. 2). A total of 5, 563 differentially expressed genes (DEGs, $\left|\log _{2}(F C)\right|>1$ ) were identified, of which 1 , 945 and 1, 824 were differentially expressed during GPA infestation in R-32 and S-27, respectively. In addition, 4,378 genes showed differential expression in the comparison of R-32 and S-27 during the 
whole experiment, suggesting the presence of differences at the transcriptional level between resistant and susceptible accessions.

DEGs during GPA infestation (from 0 to $48 \mathrm{hpi}$ ) in resistant and susceptible accessions were identified by comparing GPA treatments and the control (Fig. 4a). Notably, the DEGs were abundant at 6 hpi and 12 hpi for both R-32 and S-27. To further identify which known biological pathways were enriched with DEGs during GPA infestation, we performed KEGG pathway enrichment analysis with DEGs between the resistant and susceptible accessions in the first two days post aphid infestation (Fig. 4b-4f). Without aphid infestation, DEGs involved in 6 pathways were enriched. Among these pathways, the fold enrichment of flavonoid biosynthesis pathway was the highest. There were 21 pathways enriched with DEGs at 6 hpi. Linoleic acid metabolism pathway was highly enriched, second by brassinosteroid biosynthesis and monoterpenoid biosynthesis pathways. At $12 \mathrm{hpi}$ (15 pathways enriched), flavonoid biosynthesis pathway was significantly enriched. Seven pathways were enriched with DEGs at $24 \mathrm{hpi}$. Phenylpropanoid biosynthesis had the highest fold enrichment, followed by flavonoid biosynthesis pathway. At $48 \mathrm{hpi}$, there were 14 pathways were enriched. DEGs involved in stilbenoid, diarylheptanoid and gingerol biosynthesis pathway was highly enriched, second by flavonoid biosynthesis pathway. Except to biosynthesis of secondary metabolites pathway, flavonoid biosynthesis and phenylpropanoid biosynthesis pathways were continuously enriched by DEGs during aphid infestation. As phenylpropanoids are important precursors for the synthesis of flavonoids, this result was consistent with the accumulation of quercetin derivatives. However, the DEGs were not enriched in the terpene biosynthesis pathway. This may be due to the complexity of the terpenoid biosynthesis pathway and the unannotated biosynthesis pathway of betulin in KEGG. Therefore, the related biosynthesis pathway of betulin was not found to be enriched.

\section{Expression pattern of genes in betulin pathway during GPA infestation}

To elucidate the GPA defense mechanisms at the gene level, we investigated the expression of genes involved in the betulin biosynthesis pathway. However, the betulin biosynthesis pathway in peaches remains unclear. Based on the production process of betulin in transgenic yeast ${ }^{24}$, we selected possible genes involved in betulin biosynthesis to investigate the expression pattern of the genes at $0,6,12,24$, and 48 hpi in both R-32 and S-27. A total of 19 genes were selected, which were expressed (FPKM $>2)$ in the two genotypes at least at one time point (Fig. 5a). The relative expression of the 19 genes was normalized using logarithmic standardization. This showed that the expression of genes involved in the betulin biosynthesis pathway responded highly to GPA infestation. HMGCS (Prupe.5G088900), HMGCR2 (Prupe.8G182300), FDPS1 (Prupe.4G002700), FDPS2 (Prupe.6G028100), FDFT1 (Prupe.8G08700), SQLE3 (Prupe.8G156800), LUS1 (Prupe.3G025900), CYP716A1 (Prupe.1G002500), CYP716A3 (Prupe.4G103000) and CYP716A5 (Prupe.4G103200) were all up-regulated at $12 \mathrm{hpi}$ for both R-32 and S-27. All of these genes, apart from HMGCR2 and FDPS1, were expressed at higher levels in R-32 than in S-27 at $12 \mathrm{hpi}$. In particular, the expression of CYP716As in R-32 was consistently higher than that in S-27 during aphid infestation. In addition, the FPKMs of these CYP716As were more than twice in R-32 compared to S-27 at 
$6 \mathrm{hpi}$ and $12 \mathrm{hpi}$, which was verified by qRT-PCR (Supplementary Fig. 2). This indicated that CYP716As might have an effect on the resistance of R32 to GPA.

To further verify the function of CYP716As, we analyzed the divergence of the CYP716A subfamily in peach. The amino acid sequences of Arabidopsis thaliana CYP716As were compared with the genome sequences of $P$. persica. The other four CYP716A subfamily genes were not expressed, including CYP716A2 (Prupe.1G321100), CYP716A4 (Prupe.4G103100), CYP716A6 (Prupe.4G103300), and CYP716A7 (Prupe.6G120400), in addition to CYP716A1, CYP716A3, and CYP716A5. Fifty-five CYP716 family members from 20 plant species, including Arabidopsis thaliana, Artemisia annua, Barbarea vulgaris, Beta vulgaris, Catharanthus roseus, Coffea arabica, Lagerstroemia speciosa, Lotus japonicus, Medicago truncatula, Olea europaea, Panax notoginseng, Physcomitrella patens, Picea sitchensis, Picea glauca, Populus trichocarpa, Salvia Rosmarinus, Selaginella moellendorffii, Solanum tuberosum, Stevia rebaudiana and Vitis vinifera, were retrieved from the Cytochrome P450 Homepage ${ }^{25}$. Seven CYP716A family members in peach with the 55 CYP716 family members were performed phylogenetic analysis by comparing the amino acid sequence (Fig. $5 \mathrm{~b}$ ). As a result, CYP716A1 was divided into the clade with Medicago truncatula CYP71A12 and Lotus japonicus CYP716A51. This suggests that CYP716A1 may have a similar function to Medicago truncatula CYP71A12 and Lotus japonicus CYP716A51. CYP716A12 from Medicago truncatula has been identified as a multifunctional enzyme with the ability to catalyze consecutive oxidation at the $\mathrm{C}-28$ position of a-amyrin, $\beta$-amyrin, and lupeol, to produce ursolic, oleanolic, and betulinic acids, respectively ${ }^{24,26}$. Lotus japonicus CYP716A51 also exhibits triterpenoid C-28 oxidation activity and participates in betulinic acid biosynthesis ${ }^{27}$. Thus, CYP716A1 may play a role in betulin biosynthesis.

\section{Discussion}

There are detailed studies on aphid resistance in maize ${ }^{28}$, chrysanthemum ${ }^{29,30}$, pepper ${ }^{31}$, and others, however, studies on the defense mechanism of $P$. davidiana to aphids are relatively lagging. Although the genetics of $P$. davidiana resistance to aphids have been studied since the $1990 \mathrm{~s}^{8}$, research on the underlying molecular resistance mechanism lags far behind. This study describes the first effort in the comparative analysis of metabolomics and transcriptomics to identify the genes and metabolites involved in $P$. davidiana responses to the infestation of Myzus persicae Sülzer. The results shed light on the possible mechanisms of the dynamic responses of $P$. davidiana to Myzus persicae Sülzer.

\section{Betulin may have an effect on the resistance of $P$. davidiana to GPA}

In this study, metabolomic analysis revealed that the relative concentrations of betulin and acylglucosidate quercetin in R-32 were continuously higher than that in S-27 during aphid infestation. However, the terpene biosynthesis pathway was not enriched during aphid infestation. This may be due to the complexity of the terpenoid biosynthesis pathway and the unannotated biosynthesis pathway of betulin in KEGG. Furthermore, bioassays and field validation in the greenhouse showed that betulin had a stronger inhibitory effect on aphids than quercetin. It is reasonable to hypothesize that betulin may play a 
crucial role in the resistance of $P$. davidiana to GPA, while acylglucosidate quercetin may play a supporting role.

Betulin, a lupane-type triterpene, has received wide attention for its broad spectrum of biological activities. It has been demonstrated that betulin and betulinic acid possess antimalarial, antiinflammatory, antibacterial, antitumor, and anti-HIV properties ${ }^{32-36}$. However, studies on the anti-insect effects of betulin are rare. At the end of the last century, several studies have reported that betulin derivatives have a strong antifeedant effect against bollworm larvae (Heliothis zea) and Colorado potato beetle (Leptinotarsa decemlineata), and betulinic acid derivatives against tobacco caterpillars (Spodoptera litura F) ${ }^{37}$. Recently, betulinic acid, an insect growth regulator, has shown effective interference with the growth of Spodoptera litu ${ }^{16}$ and Callosobruchus chinensis ${ }^{17}$. This suggests that betulin may play a crucial role in the resistance of $P$. davidiana to GPA.

\section{CYP716As may play a role in regulating betulin biosynthesis}

As the identification of plant genes involved in betulin synthesis, its biosynthesis pathway in plants is becoming increasingly clear. Recent studies have revealed that betulin can be produced in yeast by catalyzing lupeol with CYP716A, a subfamily of cytochrome P450 monooxygenases ${ }^{24,38,39}$. In this study, we identified homologs of CYP716A175 24 in peach and selected three expressed CYP716As (FPKM $>1$, at least one time point), including CYP716A1, CYP716A3, and CYP716A5. The expression patterns of genes involved in the betulin biosynthesis pathway indicated that this pathway highly responded to GPA infestation, and CYP716As may be the key genes that mediate betulin biosynthesis.

As the largest enzyme family in plant metabolism, cytochrome P450 plays a crucial role in the detoxification of xenobiotics and the biosynthesis of secondary metabolites, antioxidants, and phytohormones in higher plants ${ }^{40}$. Among the cytochrome P450 superfamily, the CYP716A subfamily is mainly involved in triterpene biosynthesis ${ }^{41,42}$. Recently, Medicago truncatula CYP716A12 was identified as a multifunctional enzyme with the ability to catalyze consecutive oxidation at the $\mathrm{C}-28$ position of aamyrin, $\beta$-amyrin, and lupeol to produce ursolic, oleanolic, and betulinic acids, respectively ${ }^{24,26}$. Its homologs, Vitis vinifera CYP716A15 and CYP716A17, catalyze the oxidation of lupeol to betulin and betulinic acid ${ }^{24}$. Lotus japonicus CYP716A51 also exhibits triterpenoid C-28 oxidation activity and participates in betulinic acid biosynthesis ${ }^{27}$. Phylogenetic analysis revealed that $P$. persica CYP716A1 is clustered into a clade with Medicago truncatula CYP716A12 and Lotus japonicus CYP716A51. This implies that CYP716A1 may have a function similar to Medicago truncatula CYP716A12 and Lotus japonicus CYP716A51. Therefore, CYP716A1 may play a role in regulating betulin biosynthesis.

To investigate the molecular mechanisms of resistance to GPA in P. davidiana, integrated analyses of transcriptomic and metabolomic profiles of the resistant line R-32 and the susceptible line S-27 during the infestation of GPA were performed. The results showed a different metabolomic pattern between the resistant and sensitive peach accessions and revealed a strong toxic effect of betulin on GPA. Therefore, we proposed a model of $P$. davidiana resistance to green peach aphid (Fig. 6). Analyses of the expression 
of genes involved in betulin biosynthesis identified CYP716As as strong candidate genes regulating the expression of betulin. This study provides new insights into the prevention of aphid infestation and candidate key genes involved in resistance to GPA in $P$. davidiana.

\section{Materials And Methods}

\section{Plant materials, aphid infestation, and sampling}

Two five-year-old accessions from an $\mathrm{F}_{2}$ progeny derived from a cross between $P$. persica var. Xiangpiyoutao 1 , an aphid-susceptible nectarine, and $P$. davidiana var. Zhouxingshantao 1 (aphidresistance), were used as the plant material in this study. D29-32 was evaluated as a resistant accession (R-32) and D29-27 as a susceptible one (S-27). Trees were cultivated at the affiliated experimental orchard of ZFRI-CAAS in Xinxiang, Henan, China ( $\left.35^{\circ} 18^{\prime} \mathrm{N}, 113^{\circ} 52^{\prime} \mathrm{E}\right)$. Standard agricultural practices were adopted, except for experimental treatments.

For aphid infestation, treatment was performed at two weeks (pesticide duration) after spraying the insecticide Closer (Dow AgroSciences, USA). A total of 15 adult GPAs (Myzus persicae Sülzer), collected from a naturally infested peach orchard (Zhengzhou Fruit Research Institute, Zhengzhou, China), were placed on the partially expanded trifoliate leaves of each shoot for each individual and subsequently covered with an argenteus fly net. Shoot tips with young leaves were sampled at time 0 (no aphid infestation), 6, 12, 24, and $48 \mathrm{~h}$ post infestation (hpi). Before sampling, the remaining aphids were carefully removed from the leaves. The collected shoot materials were immediately frozen in liquid nitrogen and stored at $-80^{\circ} \mathrm{C}$. Two biological replicates were used for each sampling point.

\section{RNA isolation, mRNA sequencing, and RNA-Seq Data Analysis}

The same samples used for the metabolome analyses were also used to perform RNA-sEq. Total RNA was extracted using the Quick RNA Isolation Kit (Huayueyang Biotechnology Co., Ltd., Beijing, China) according to the manufacturer's instructions. RNA purity and RNA integrity were examined using the kaiaoK5500®Spectrophotometer (Kaiao, Beijing, China), and the concentration was confirmed using the RNA Nano 6000 Assay Kit of the Bioanalyzer 2100 system (Agilent Technologies, CA, USA). A total of 24 sequencing libraries (two biological replicates at six time points for both the aphid-resistant andsusceptible lines) were created from $2 \mu$ g RNA per sample using the NEBNext ${ }^{\circledR}$ Ultra ${ }^{\text {TM }}$ RNA Library Prep Kit for Illumina ${ }^{\circledR}$ (\#E7530L, NEB, USA) following the manufacturer's recommendations. RNA concentration of libraries was measured using Qubit ${ }^{\circledR}$ RNA Assay Kit in Qubit ${ }^{8}$ 3.0, and each library's quality was then assessed using the Agilent Bioanalyzer 2100 system (Agilent Technologies).

Reference peach genome v2.0 and annotation files were downloaded from the GDR website ( https://www.rosaceae.org/species/prunus_persica/genome_v2.0.a1) ${ }^{43}$. The genome index was built using Bowtie2 v2.2.3 ${ }^{44}$. Clean data (clean reads) were obtained by trimming Smart-seq $2^{45}$ public primer sequences and removing reads containing adapters, reads with more than $5 \%$ unknown nucleotides, and low-quality reads from raw data. The clean data were then aligned to the reference genome using HISAT2 
v2.1.0 ${ }^{46}$ with default parameters. To estimate the expression levels of genes in each sample, the read numbers for each gene in each sample were counted by HTSeq $v 0.6 .0^{47}$, and then normalized to fragments per kilobase million mapped reads (FPKM). Sequencing data in this study were deposited in the NCBI Short Read Archive (SRA) under the accession PRJNA692810.

\section{Validation by quantitative real-time PCR}

To validate the RNA-seq data, the expression levels of 10 DEGs were evaluated by qRT-PCR using the cDNA samples used for RNA-Seq library construction as templates. Gene-specific primers (Supplementary Table 4) were designed using a primer-blast program from NCBI (http://www.ncbi.nlm.nih.gov/tools/primer-blast/). qRT-PCR was performed using the Roche Light Cycler 480 (Roche, Basel, Switzerland) at the following conditions: $95^{\circ} \mathrm{C}$ for $30 \mathrm{~s}$, followed by 45 cycles at $95^{\circ} \mathrm{C}$ for $10 \mathrm{~s}, 57^{\circ} \mathrm{C}$ for $10 \mathrm{~s}$, and $72^{\circ} \mathrm{C}$ for $15 \mathrm{~s}$. Actin was used as a housekeeping gene to normalize the amplification. The relative transcript levels for each sample were calculated using the $2^{-\Delta \Delta \mathrm{Ct}}$ method.

\section{Metabolome analyses}

A total of 20 samples, including two biological replicates at five time points, were used for metabolomic analysis. Sample preparation and UPLC-MS/MS (UPLC, Shim-pack UFLC SHIMADZU CBM30A system, www.shimadzu.com.cn/; MS, Applied Biosystems4500 Q TRAP, www.appliedbiosystems.com.cn/) was

performed as described previously ${ }^{48}$. Quality control (QC) samples were prepared by mixing extracts from samples to analyze the repeatability. During instrumental analysis, a quality control sample was inserted into every 10 sample analyses to monitor the repeatability of the analysis process. Based on the self-built database MWDB (Metware database); the metabolites of the samples were qualitatively and quantitatively analyzed by mass spectrometry data obtained using Analyst 1.6.1 software. According to the secondary spectrum information, isotope signals, including repeated signals of $\mathrm{K}^{+}, \mathrm{N}^{+}$, and $\mathrm{NH}_{4}{ }^{+}$ions, and fragment ions of other larger molecular weight substances were removed during the analysis.

Significantly differentially accumulated metabolites (DAMs) between groups were determined by VIP $\geq 1$ and absolute $\log _{2} \mathrm{FC}$ (fold change) $\geq 1$. VIP values were extracted from orthogonal projections to latent structures-discriminant analysis (OPLS-DA) results, which also contain score plots, and permutation plots were generated using the $R$ package MetaboAnalyst $R^{49}$. The data were log-transformed $\left(\log _{2}\right)$ and meancentered before the OPLS-DA. To avoid overfitting, a permutation test (200 permutations) was performed.

\section{Bioassays and field validation in the greenhouse}

The toxicity of the selected metabolites (betulin and quercetin) to GPA was measured using the slip-dip method according to Zhou et al ${ }^{50}$. Five hundred and forty Apterous adult GPAs were used as test insects for each compound. The compounds were dissolved in a solution of $3 \%$ acetone containing $0.1 \%$ Tween 80 . Six doses $(2,1,0.5,0.25,0.125$, and $0.0625 \mathrm{~g} / \mathrm{mL}$ ) were set with three replicates. Tobacco (Nicotiana tabacum) leaf discs $(1 \times 1 \mathrm{~cm})$ were added to the corresponding solutions for $5 \mathrm{~s}$, removed, and dried. Tobacco leaf discs treated with a solution of $3 \%$ acetone containing Tween 80 alone were used as the blank control (CT). The aphids were observed after $24 \mathrm{~h}$ and $48 \mathrm{~h}$ of rearing under controlled growth 
conditions, as described above. Aphids that exhibited immobility or irregularly trembling legs were considered dead. The lethal concentrations for subsequent experiments were determined based on logprobit analysis of concentration-mortality data.

The inhibitory effect of betulin against aphids was evaluated under greenhouse conditions. S-27 shoots, sprayed with betulin (396.08 $\mathrm{mg} \cdot \mathrm{L}^{-1}, 24 \mathrm{~h} \mathrm{LC}{ }_{50}$ ) or acetone (as CK), were used as test plant materials. Each treatment consisted of three shoots. Every shoot was infested with 15 adult GPAs after it was sprayed with the corresponding solutions and dried. The number of GPAs in the shoots was recorded daily for a week. The experiment was carried out at $25 \pm 1{ }^{\circ} \mathrm{C}, 60-80 \% \mathrm{RH}$, and a $14: 10 \mathrm{~h}$ light/dark photoperiod.

\section{Declarations}

Data availability. All data related to this paper is available upon request.

\section{Acknowledgement}

This study was supported by the National Key Research and Development Program (2019YFD1000200); and the Agricultural Science and Technology Innovation Program (CAAS-ASTIP-2018-ZFRI-01).

\section{Author contributions}

L. W. conceived and supervised the study. J. W. performed the aphid infestation and RNA-seq experiments and analyzed the data. J. W. and Y. L. wrote the manuscript. All authors read and approved the final manuscript.

\section{Additional information}

Supplementary information is available for this paper at

Competing interests: The authors declare no competing financial interests.

Reprints and permission information is available online at

\section{References}

1. Mickelbart, M. V., Hasegawa, P. M. \& Bailey-Serres, J. Genetic mechanisms of abiotic stress tolerance that translate to crop yield stability. Nature Reviews Genetics. 16, 237-251 (2015).

2. Massonié, G., Maison, P., Monet, R. \& Grasselly, C. Résistance au puceron vert du pêcher, Myzus persicae Sulzer (Homoptera Aphididae) chez Prunus persica (L.) Batsch et d'autres espèces de Prunus. Agronomie. 2, 63-70 (1982).

3. Monet, R. \& Massonié, G. Déterminisme génétique de la résistance au puceron vert (Myzus persicae) chez le pêcher. Résultats complémentaires. Agronomie. 14, 177-182 (1994). 
4. Sauge, M. H., Kervella, J. \& Rahbé, Y. Probing behaviour of the green peach aphid Myzus persicae on resistant Prunus genotypes. Entomologia Experimentalis et Applicata. 89, 223-232 (1998).

5. Niu, L. et al. Inheritance analysis of resistance to green peach aphids (Myzus persicae Sülzer) for peach cultivar 'Fen Shouxing' (Prunus persica var. densa). Journal of Fruit Science. 33, 578-84 (2016).

6. Niu, L. et al. Dynamic transcriptomes of resistant and susceptible peach lines after infestation by green peach aphids (Myzus persicae Sülzer) reveal defence responses controlled by the Rm3 locus. BMC Genomics. 19, 846 (2018).

7. Wang, L., Zhu, G., Fang, W., Zuo, T. \& Han, L. Study on the resistance to peach aphid (Myzus persicae Sulzer) of peach germplasm. Journal of Fruit Science. 18, 145-147 (2001).

8. Sauge, M. H., Kervella, J. \& Pascal, T. Settling behaviour and reproductive potential of the green peach aphid Myzus persicae on peach varieties and a related wild Prunus. Entomologia Experimentalis Et Applicata. 89, 233-242 (1998).

9. Guo, J. et al. The Asian corn borer Ostrinia furnacalis feeding increases the direct and indirect defence of mid-whorl stage commercial maize in the field. Plant Biotechnol J. 17, 88-102 (2019).

10. Qi, J. et al. Oral secretions from Mythimna separata insects specifically induce defence responses in maize as revealed by high-dimensional biological data. Plant, Cell \& Environment. 39, 1749-1766 (2016).

11. Jakobs, R., Schweiger, R. \& Müller, C. Aphid infestation leads to plant part-specific changes in phloem sap chemistry, which may indicate niche construction. New Phytologist. 221, 503-514 (2019).

12. Sauge, M. H., Lambert, P. \& Pascal, T. Co-localisation of host plant resistance QTLs affecting the performance and feeding behaviour of the aphid Myzus persicae in the peach tree. Heredity. 108, 292-301 (2012).

13. Selin-Rani, S. et al. Toxicity and physiological effect of quercetin on generalist herbivore, Spodoptera litura Fab. and a non-target earthworm Eisenia fetida Savigny. Chemosphere. 165, 257-267 (2016).

14. Damestoy, T. et al. Oak genotype and phenolic compounds differently affect the performance of two insect herbivores with contrasting diet breadth. Tree physiology. 39, 615-627 (2019).

15. Ma, T., Shi, X., Ma, S., Ma, Z. \& Zhang, X. Evaluation of physiological and biochemical effects of two Sophora alopecuroides alkaloids on pea aphids Acyrthosiphon pisum. Pest management science. 76, 4000-4008 (2020).

16. Badathu, M., Lingampally, V. \& Kaur, A. Effect of betulinic acid on Spodoptera litura (Fab.) (Lepidoptera: Noctuidae). Indian J. Sci. Res. 8, 177-181 (2014).

17. Madhavi, M., Babu Rao, G. \& Srinivas, V. Morphological abnormalities of betulinic acid from Ziziphus Jujuba against the Callasobruchus chinensis (Coleoptera: Bruchidae). Biosciences Biotechnology Research Asia. 16, 411-416 (2019).

18. Lattanzio, V., Arpaia, S., Cardinali, A., Di Venere, D. \& Linsalata, V. Role of endogenous flavonoids in resistance mechanism of Vigna to aphids. Journal of Agricultural and Food Chemistry. 48, 5316$5320(2000)$. 
19. Sharma, R. \& Sohal, S. K. Bioefficacy of quercetin against melon fruit fly. Bulletin of Insectology. 66, 79-83 (2013).

20. Goławska, S., Sprawka, I., Łukasik, I. \& Goławski, A. Are naringenin and quercetin useful chemicals in pest-management strategies? Journal of pest science. 87, 173-180 (2014).

21. Yu, X. H., Chen, M. H. \& Liu, C. J. Nucleocytoplasmic-localized acyltransferases catalyze the malonylation of 7-0-glycosidic (iso)flavones in Medicago truncatula. Plant J. 55, 382-396 (2008).

22. Koirala, N., Pandey, R. P., Thang, D. V., Jung, H. J. \& Sohng, J. K. Glycosylation and subsequent malonylation of isoflavonoids in E. coli: strain development, production and insights into future metabolic perspectives. J Ind Microbiol Biotechnol. 41, 1647-1658 (2014).

23. Verde, I. et al. The Peach v2. 0 release: high-resolution linkage mapping and deep resequencing improve chromosome-scale assembly and contiguity. Bmc Genomics. 18, 225 (2017).

24. Fukushima, E. O. et al. CYP716A subfamily members are multifunctional oxidases in triterpenoid biosynthesis. Plant and Cell Physiology. 52, 2050-2061 (2011).

25. Nelson, D. R. The cytochrome P450 homepage. Human Genomics. 4, 59-65 (2009).

26. Carelli, M. et al. Medicago truncatula CYP716A12 is a multifunctional oxidase involved in the biosynthesis of hemolytic saponins. Plant Cell. 23, 3070-3081 (2011).

27. Suzuki, H. et al. Lotus japonicus triterpenoid profile and characterization of the CYP716A51 and LjCYP93E1 genes involved in their biosynthesis in planta. Plant \& cell physiology. 60, 2496-2509 (2019).

28. Varsani, S. et al. 12-oxo-phytodienoic acid acts as a regulator of maize defense against corn leaf aphid. Plant Physiol. 179, 1402-1415 (2019).

29. An, C. et al. Overexpression of CmMYB15 provides chrysanthemum resistance to aphids by regulating the biosynthesis of lignin. Hortic Res. 6, 84-84 (2019).

30. Zhang, W. et al. Chrysanthemum CmWRKY53 negatively regulates the resistance of chrysanthemum to the aphid Macrosiphoniella sanborni. Hortic Res. 7, 109 (2020).

31. Sun, M., Voorrips, R. E., Kaauwen, M. V., Visser, R. G. F. \& Vosman, B. The ability to manipulate ROS metabolism in pepper may affect aphid virulence. Hortic Res. 7, 6 (2020).

32. Moghaddam, M. G., Ahmad, F. B. H. \& Samzadeh-Kermani, A. Biological activity of betulinic acid: a review. Pharmacology \& Pharmacy. 3, 119-123 (2012).

33. Zhao, H., Liu, Z., Liu, W., Han, X. \& Zhao, M. Betulin attenuates lung and liver injuries in sepsis. International immunopharmacology. 30, 50-56 (2016).

34. Jonnalagadda, S. C., Suman, P., Morgan, D. C. \& Seay, J. N. Recent developments on the synthesis and applications of betulin and betulinic acid derivatives as therapeutic agents. Studies in Natural Products Chemistry. 53, 45-84 (2017).

35. Hordyjewska, A., Ostapiuk, A. \& Horecka, A. Betulin and betulinic acid in cancer research. Journal of Pre-Clinical and Clinical Research. 12, $72-75$ (2018). 
36. Huang, Q. X. et al. Structure and anti-HIV activity of betulinic acid analogues. Current medical science. 38, 387-397 (2018).

37. Jagadeesh, S. G., Krupadanam, G. L. D., Srimannarayana, G., Murthy, S. S., Kaur, A. \& Raja, S. S. Tobacco Caterpillar Antifeedent from the Gotti Stem Wood Triterpene Betulinic Acid. Journal of Agricultural \& Food Chemistry. 46, 2797-2799 (1998).

38. Huang, L. et al. Molecular characterization of the pentacyclic triterpenoid biosynthetic pathway in Catharanthus roseus. Planta. 236, 1571-1581 (2012).

39. Sun, J. et al. Glycerol improves heterologous biosynthesis of betulinic acid in engineered Yarrowia lipolytica. Chemical Engineering Science. 196, 82-90 (2019).

40. Pandian, B. A., Sathishraj, R., Djanaguiraman, M., Prasad, P. V. V. \& Jugulam, M. Role of cytochrome P450 enzymes in plant stress response. Antioxidants. 9, 454 (2020).

41. Misra, R. C. et al. Two CYP716A subfamily cytochrome P450 monooxygenases of sweet basil play similar but nonredundant roles in ursane- and oleanane-type pentacyclic triterpene biosynthesis. New Phytologist. 214, 706-720 (2017).

42. Moses, T. et al. OSC2 and CYP716A14v2 catalyze the biosynthesis of triterpenoids for the cuticle of aerial organs of Artemisia annua. Plant Cell. 27, 286-301 (2015).

43. Verde, I. et al. The high-quality draft genome of peach (Prunus persica) identifies unique patterns of genetic diversity, domestication and genome evolution. Nature genetics. 45, 487-494 (2013).

44. Langmead, B. \& Salzberg, S. L. Fast gapped-read alignment with Bowtie 2. Nature methods. 9, 357359 (2012).

45. Picelli, S. et al. Full-length RNA-seq from single cells using Smart-seq2. Nature protocols. 9, 171-181 (2014).

46. Kim, D., Langmead, B. \& Salzberg, S. L. HISAT: a fast spliced aligner with low memory requirements. Nature methods. 12, 357-360 (2015).

47. Anders, S., Pyl, P. T. \& Huber, W. HTSeq-a Python framework to work with high-throughput sequencing data. Bioinformatics. 31, 166-169 (2015).

48. Ding, X. et al. Metabolomics studies on cytoplasmic male sterility during flower bud development in soybean. International Journal of Molecular Sciences. 20, 2869 (2019).

49. Chong, J. \& Xia, J. MetaboAnalystR: an R package for flexible and reproducible analysis of metabolomics data. Bioinformatics. 34, 4313-4314 (2018).

50. Zhou, H. et al. Acaricidal mechanism of scopoletin against Tetranychus cinnabarinus. Frontiers in Physiology. 10, 164 (2019).

\section{Figures}


$\mathbf{a}$
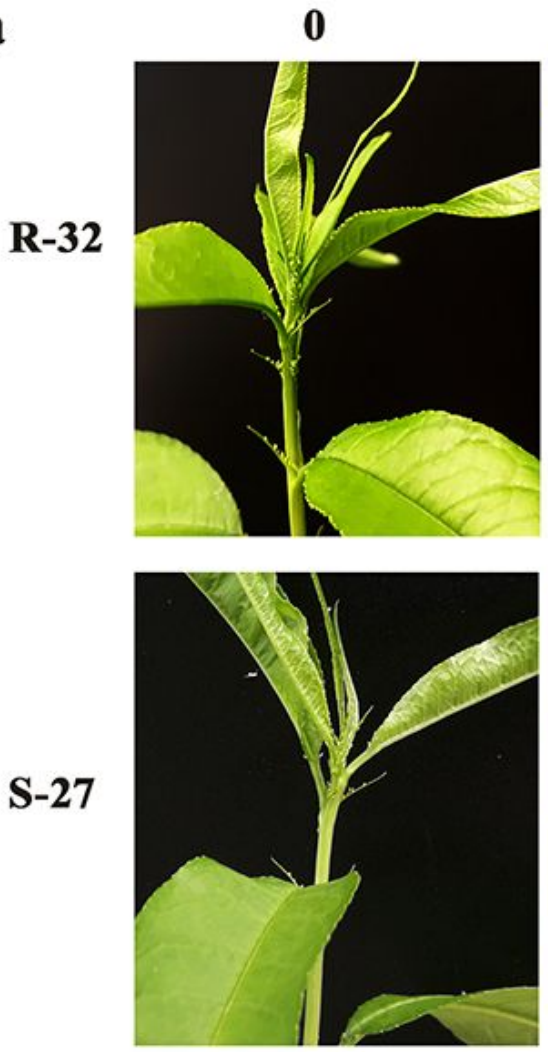

1 DPI
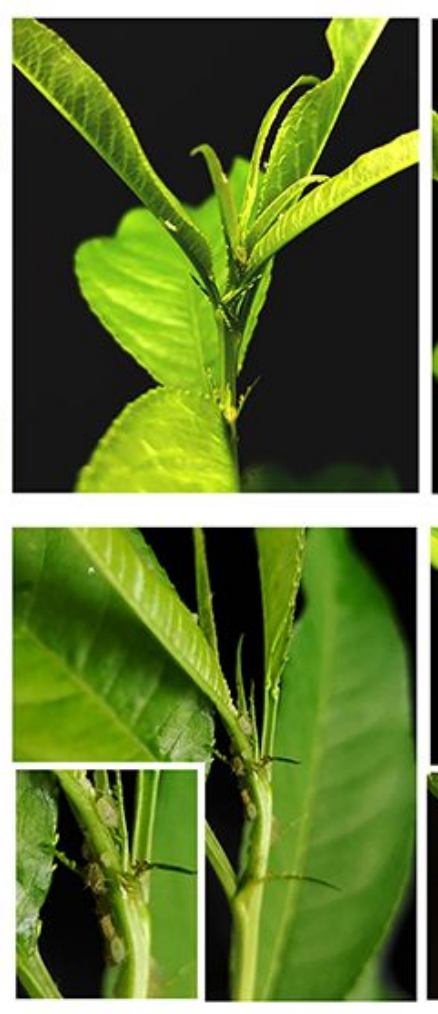

2 DPI
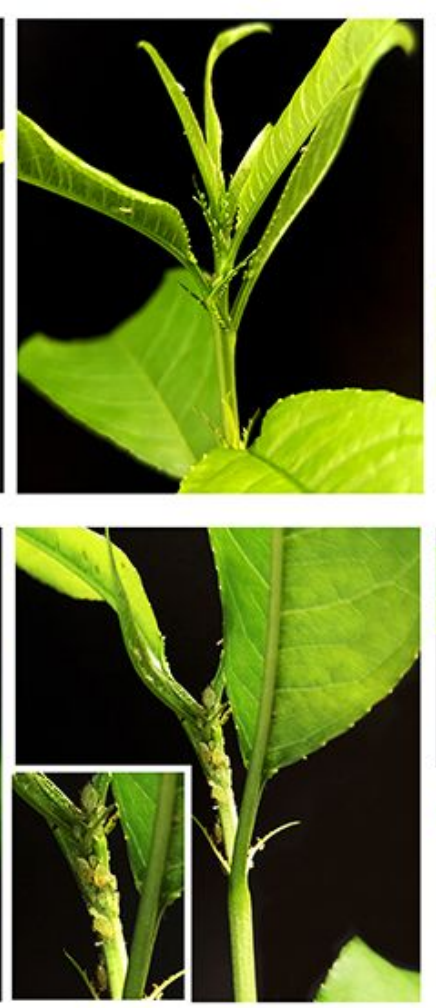

5 DPI
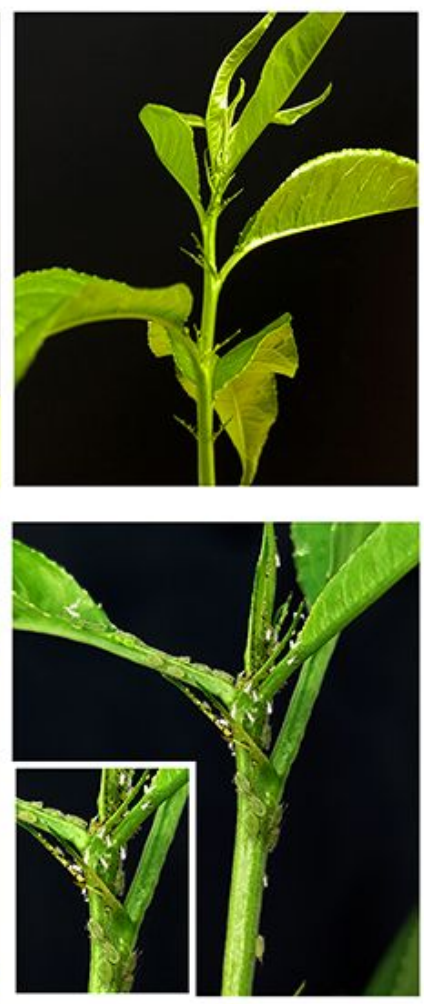

b

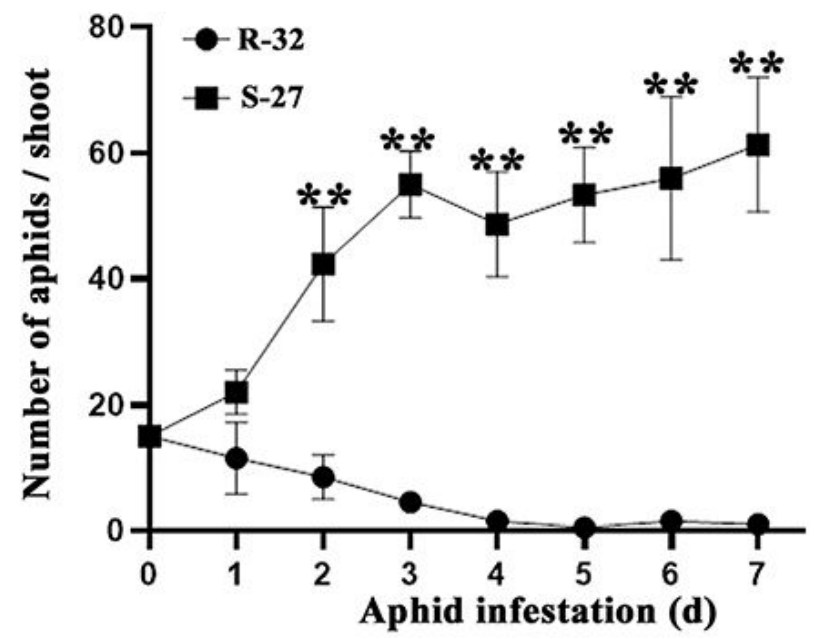

c

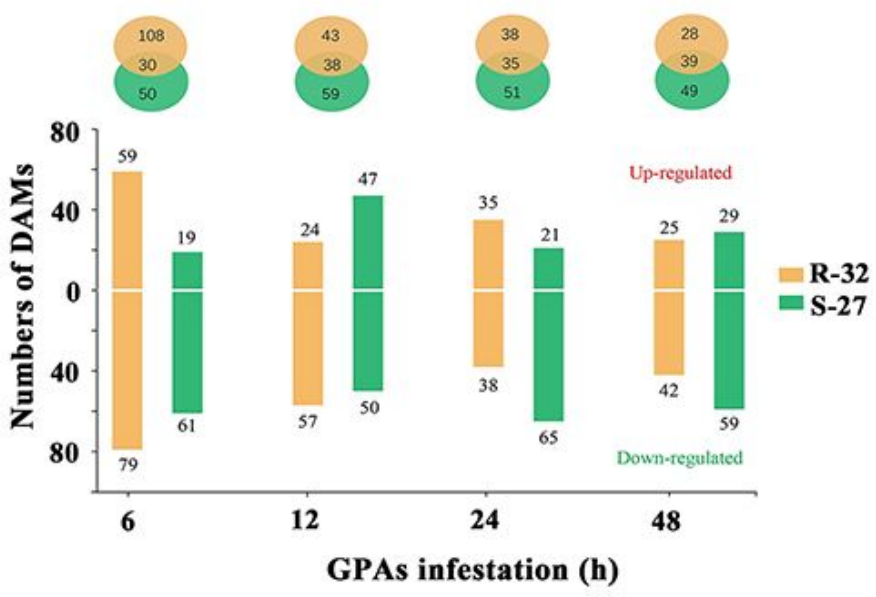

Figure 1

Different resistance of R-32 and S-27 to GPA. (a) Pictures of GPA settlement on both R-32 (resistant) and S-27 (susceptible). 0 represents the control non-infested samples for both R-32 and S-27. 1, 2 and 3 DPI refer to 1-, 2- and 3-days post aphid infestation for R-32 and S-27. Small pictures in the left bottom are magnification for each corresponding picture. (b) The number of aphids on shoots of R-32 and S-27. (c) The up- and down-regulated differentially accumulated metabolites (DAMs) in R-32 and S-27 between GPA treatments and control. The DAMs that appeared in both samples are represented by the overlapping regions of the Venn diagram. Light orange and light green represent the DAMs in R-32 and S-27, respectively. 
a
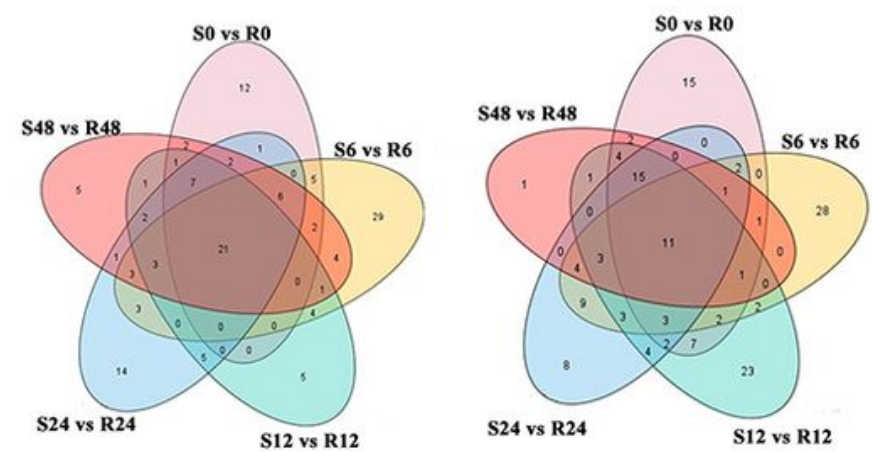

Up-regulated DAMs
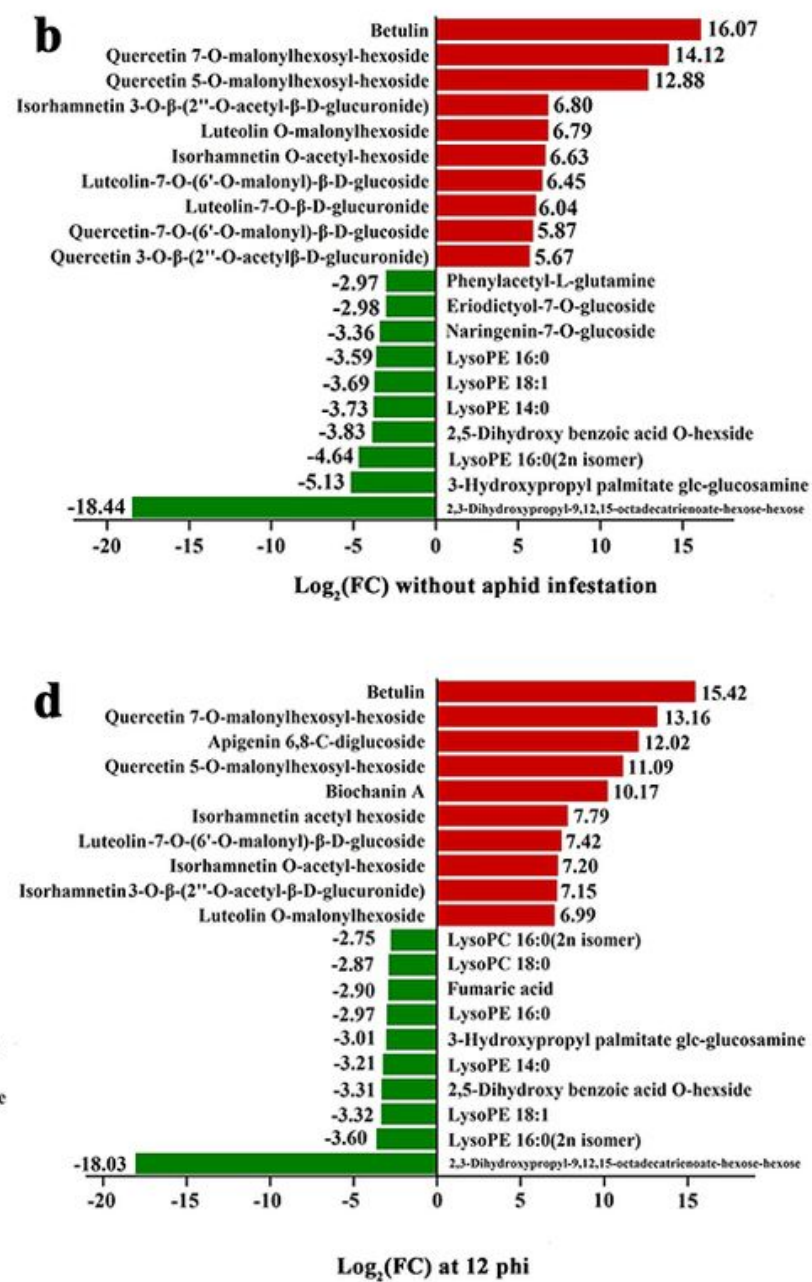

$\log _{2}(\mathrm{FC})$ at $12 \mathrm{phi}$

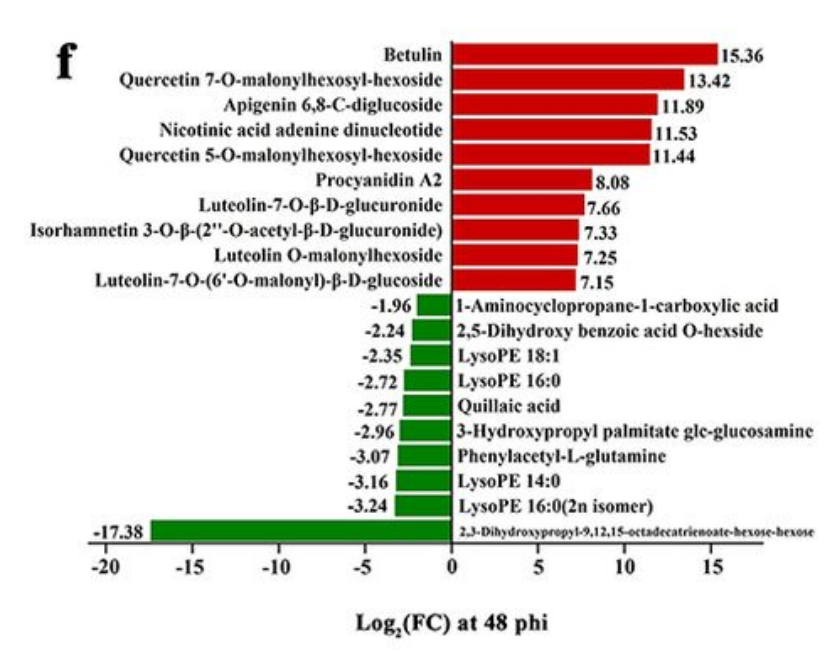

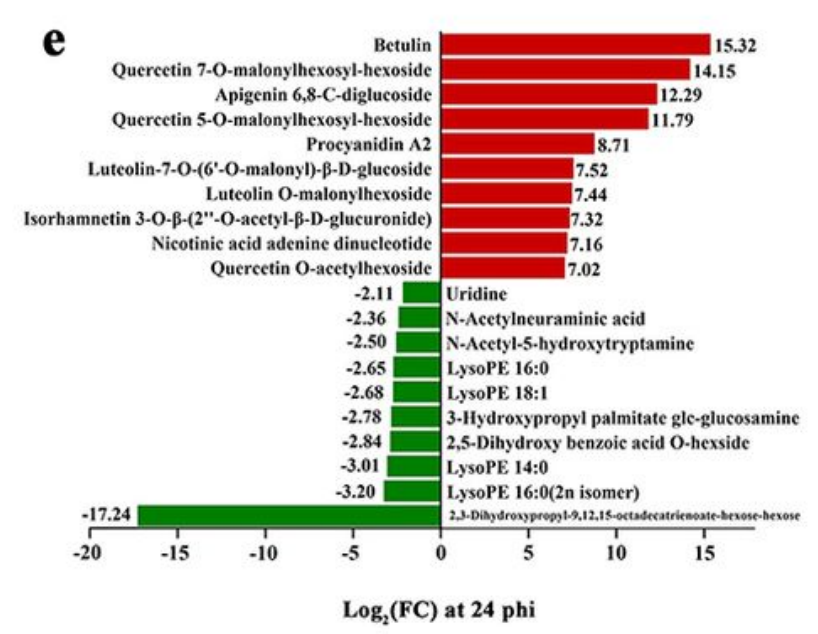

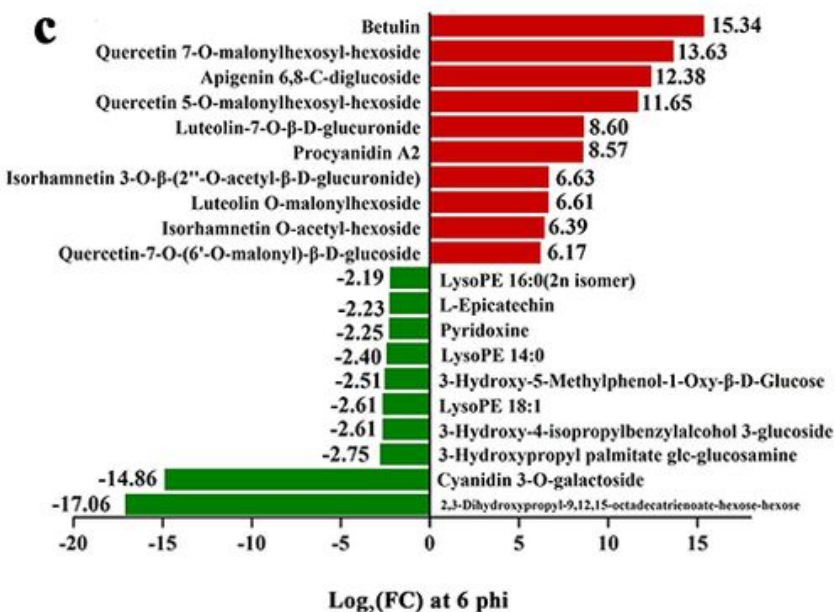

Figure 2

Differentially Accumulated Metabolites (DAMs) between R-32 and S-27. (a) The venn diagrams of upregulated DAMs (left) and down-regulated DAMs (right) for R-32 compared to S-27 at 0, 6-, 12-, 24- and 48-hpi. (b) Top twenty up- and down-regulated DAMs in R-32, compared to S-27, without aphid infestation. (c-f) Top twenty up- and down-regulated DAMs in R-32 at 6 hpi (c), 12 hpi (d), 24 hpi (e) and 48 hpi (f) compared to S-27. 

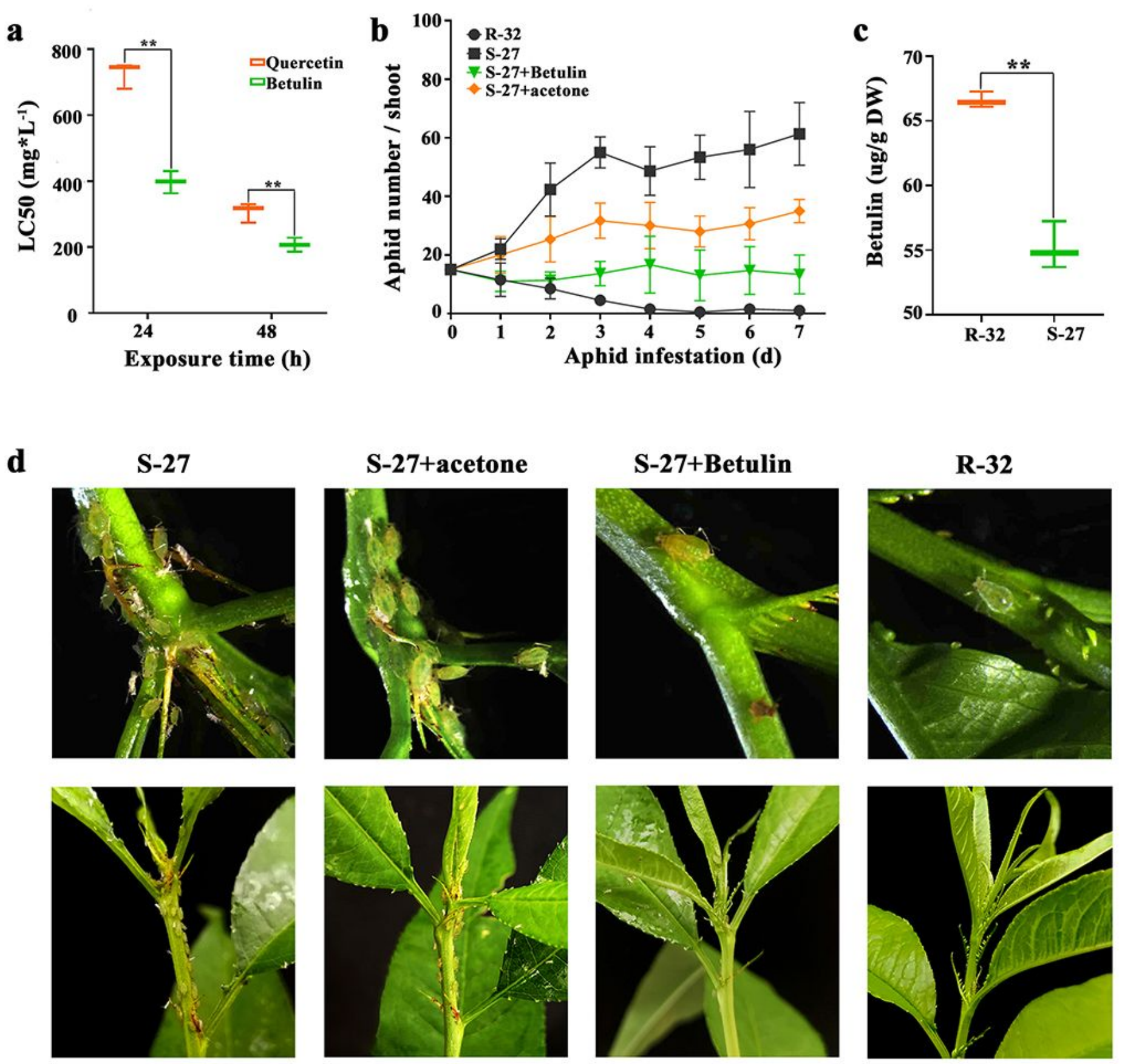

Figure 3

The toxicity of betulin to GPA. (a) The median lethal concentration (LC50) values of betulin and quercetin at $24 \mathrm{~h}$ and $48 \mathrm{~h}$ of exposure. (b) The number of aphids on shoots. S-27+acetone, S-27 sprayed with acetone; S-27+Betulin, S-27 sprayed with 396.08 mgL-1 betulin. (c) The absolute content of betulin in R32 and S-27. (d) Representative pictures of aphid settlement after aphid infestation for 7 days. Above pictures were the magnification for below pictures, correspondingly. Above figures were taken by stereo microscope and below pictures were taken in the greenhouse by camera. 
a

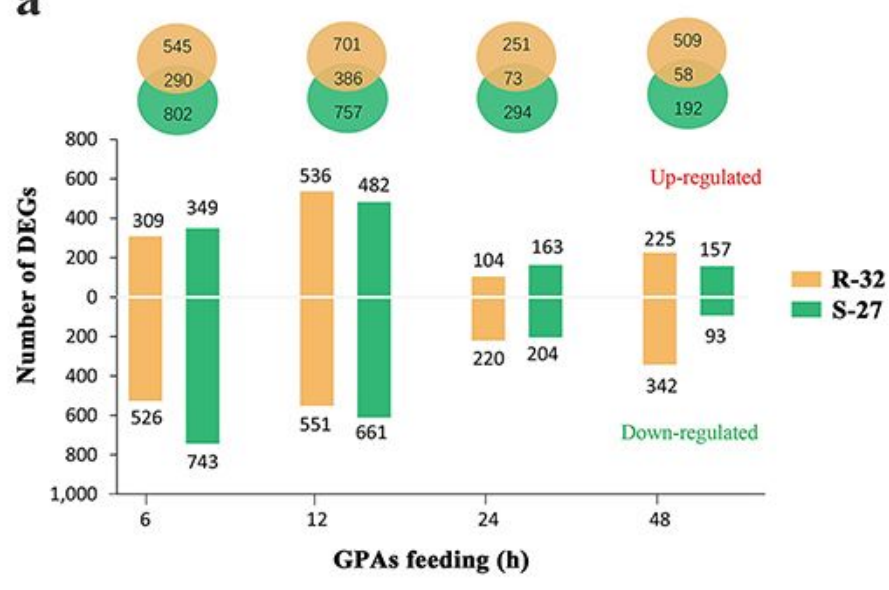

b

Biosynthesis of secondary metabolites

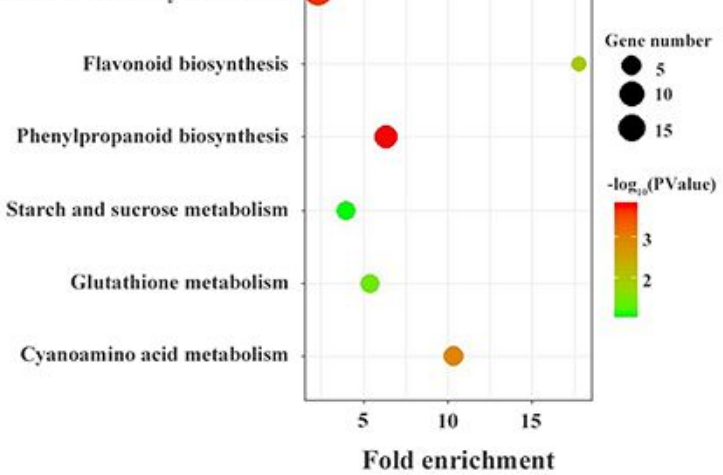

Enriched pathway without aphid infestation

d

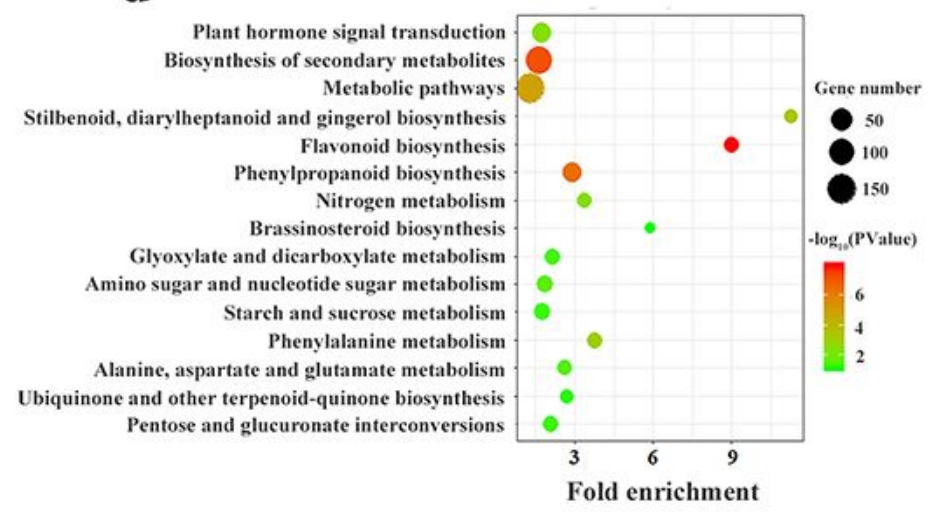

Enriched pathway at $12 \mathrm{hpi}$

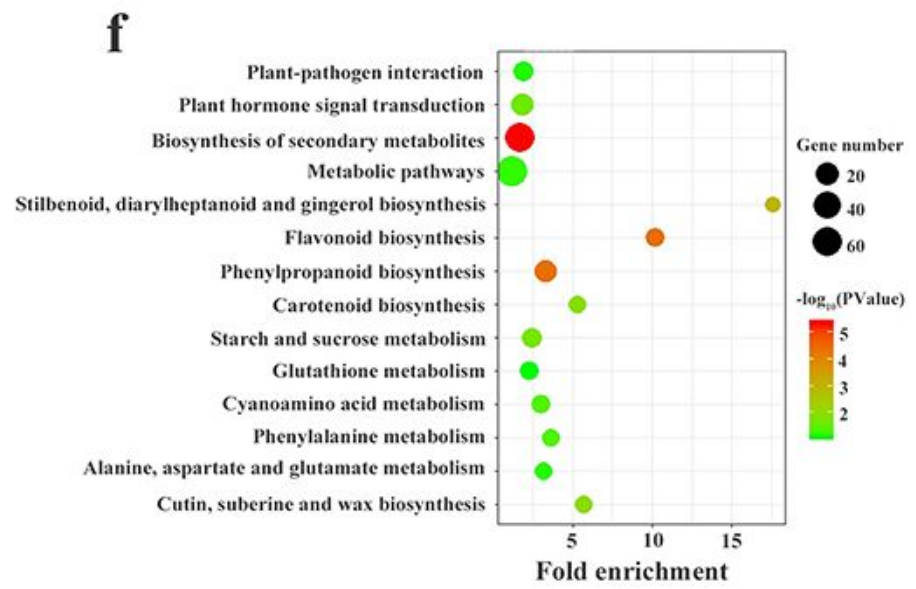

Enriched pathway at $48 \mathrm{hpi}$

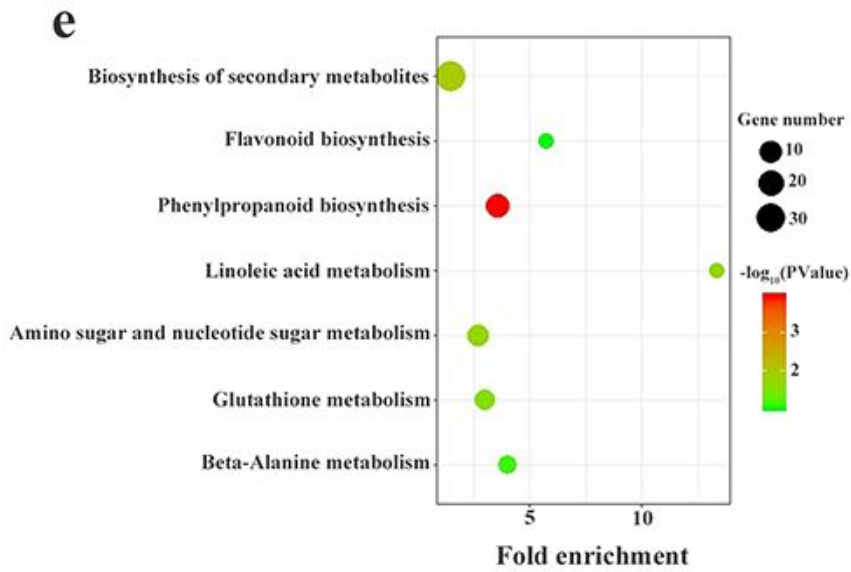

Enriched pathway at $24 \mathrm{hpi}$ . c Plant hormone signal transduction
Biosynthesis of secondary metabolites Metabolic pathways
Flavonoid biosynthesis propanoid biosynthesis. Sesquiterpenoid and triterpenoid biosynthesis Brassinosteroid biosynthesis Diterpenoid biosynthesis Linoleic acid metabolism Glycerolipid metabolism nucleotide sugar metabolism Starch and sucrose metabolism Cyanoamino acid metabolism
Phenylalanine, tyrosine and tryptophan biosynthesis
Tyrosine metabolism Fatty acid degradation Pentose and glucuronate interconversions Enriched pathway at $6 \mathrm{hpi}$

Fold enrichment 
and S-27. $Y$ axis, KEGG pathway terms. X-axis, fold enrichment. Fold enrichment $=(m / M) /(n / N)$, where $m$ denotes the number of "hits" (mapped genes) in the pathway, $M$ denotes the number of genes in the pathway, $\mathrm{n}$ denotes the number of "hits" in the background and $\mathrm{N}$ denotes the number of total genes in the background.

a

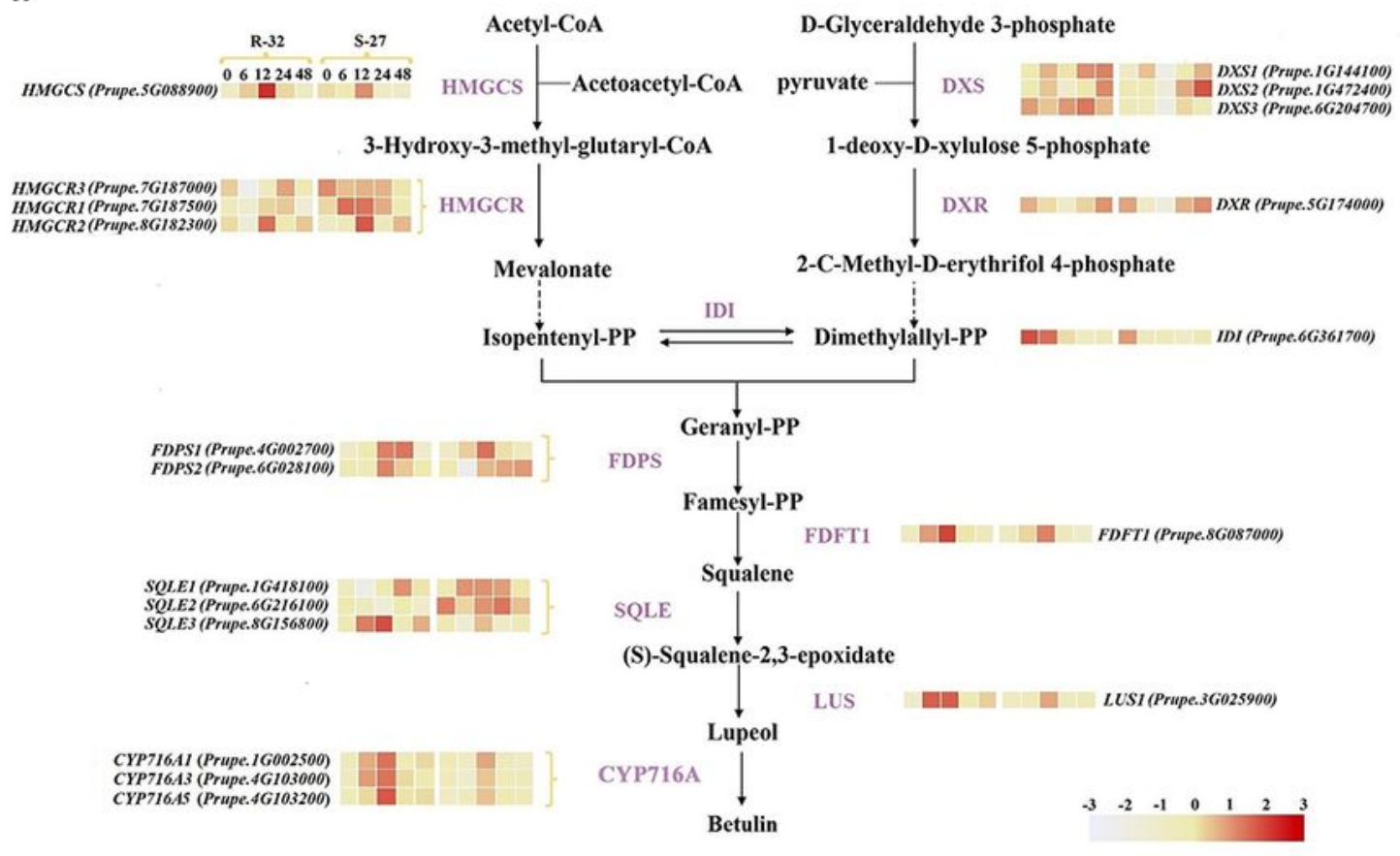

b

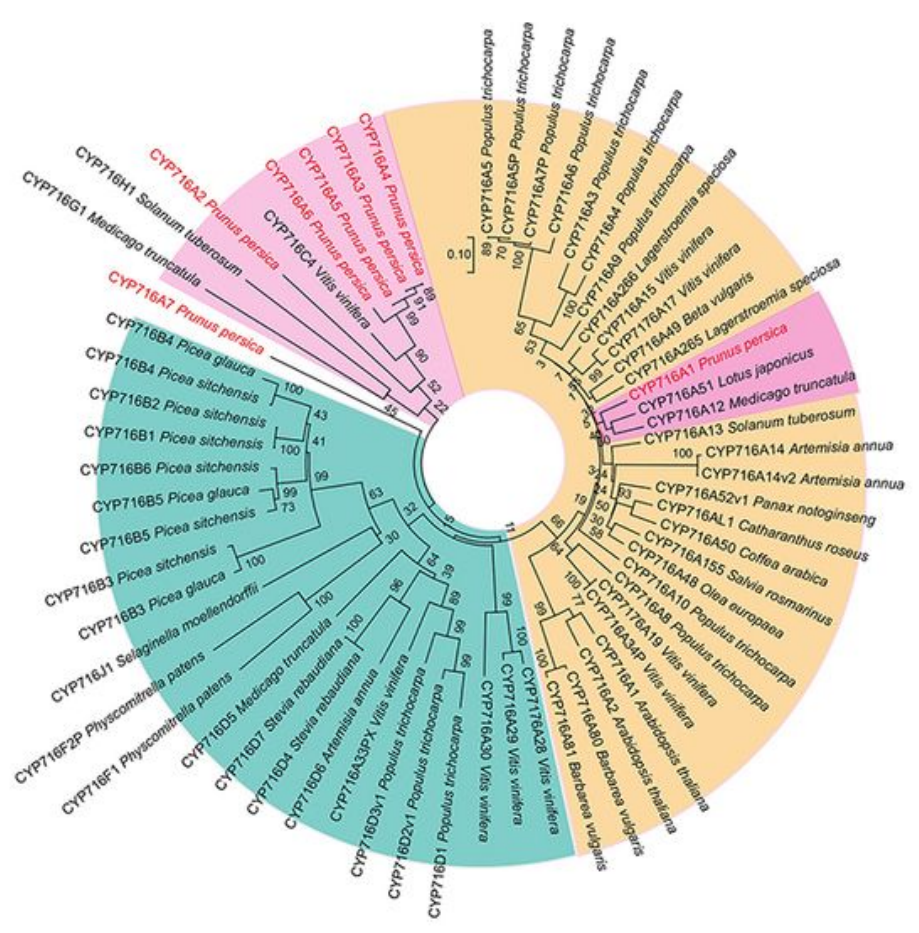

Figure 5 
The function of CYP716As in regulating betulin biosynthesis. (a) Expression patterns of 19 genes involved in betulin biosynthesis pathway at $0,6,12,24$, and 48 hpi in R-32 and S-27. Purple abbreviations refer to enzymes and the corresponding KEGG IDs and enzyme names are shown in Table S6. 0 represents the control non-infested samples for R-32 and S-27. 6 (6 hpi), 12 (12 hpi), 24 (24 hpi) and 48 (48hpi) refer to 6, 12, 24 and $48 \mathrm{~h}$ after aphid infestation for R-32 and S-27. (b) A phylogenetic tree derived from amino acid sequences of CYP716As in plants. Alignment of full amino acid sequences of CYP716As was performed by MUSCLE software, and the phylogenetic tree was constructed using Neighbor-Joining method by MEGA software (v7.0). Numbers indicate bootstrap test results with 1000 replicate analyses. CYP716 family member sequences were retrieved from the Cytochrome P450 Homepage.

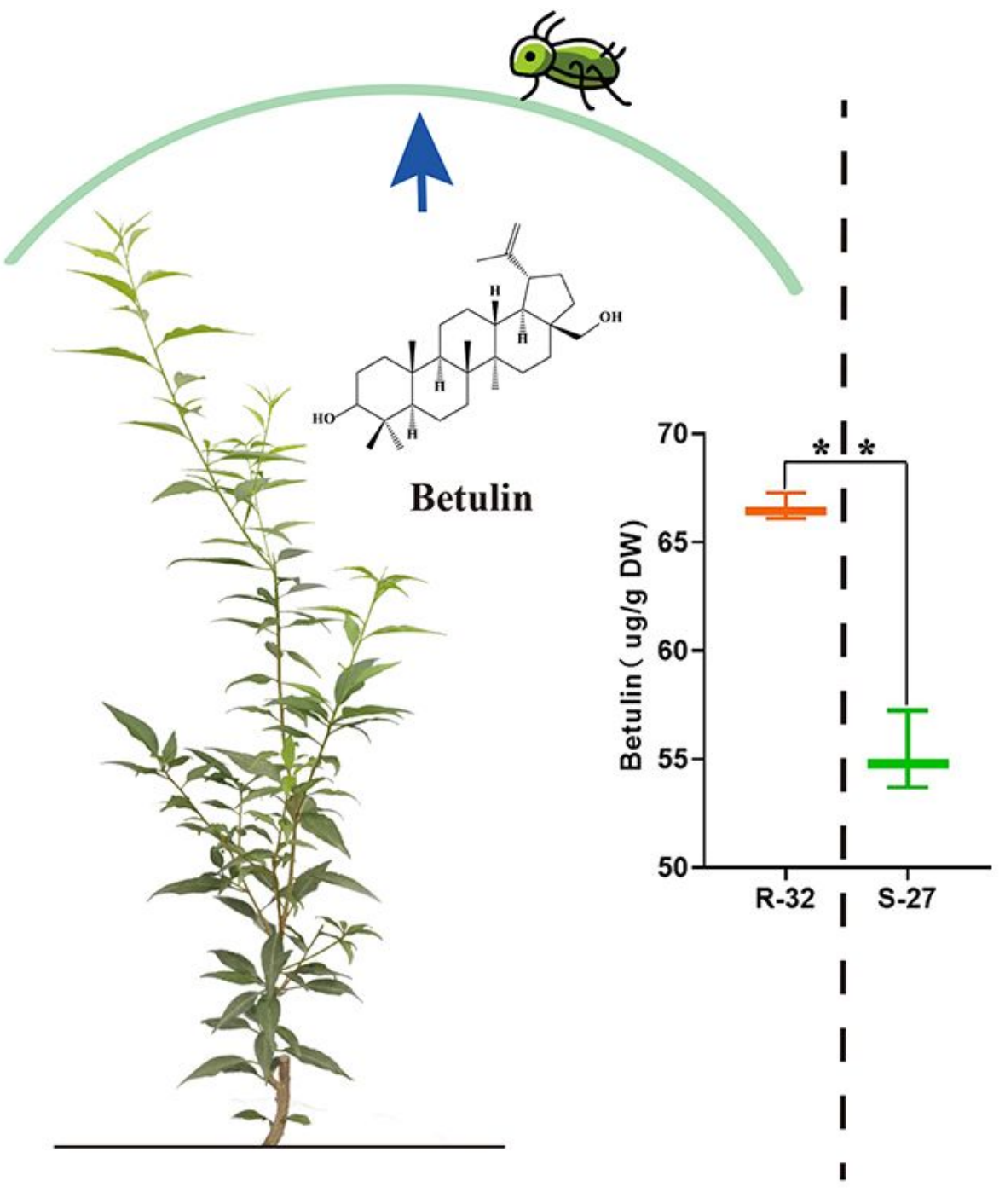

R-32

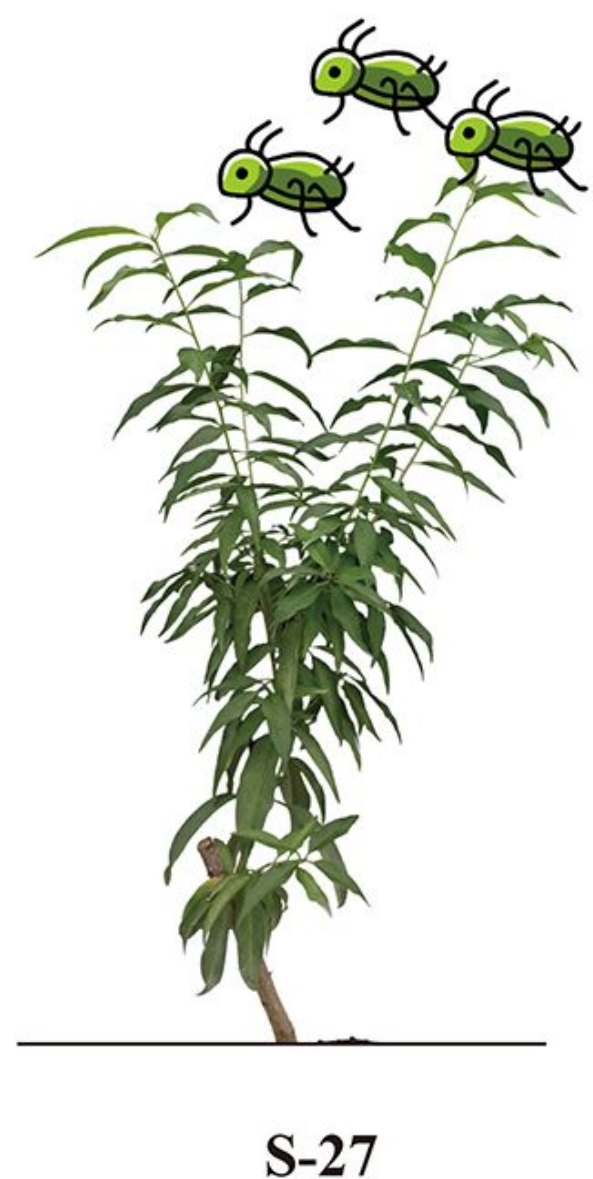

Figure 6

A model of P. davidiana resistance to green peach aphid.

\section{Supplementary Files}


This is a list of supplementary files associated with this preprint. Click to download.

- SupplementaryTable1.xlsx

- SupplementaryTable2.xlsx

- SupplementaryTable3.xlsx

- supplementaryFigures.docx 